\title{
Molecular survey of duck circovirus infection in poultry in southern and southwestern China during 2018 and 2019
}

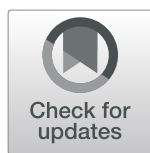

Hao Liu' ${ }^{1 \dagger}$, Li Xia Li ${ }^{1,2 \dagger}$, Wen Chao Sun ${ }^{3 \dagger}$, Ning Shi ${ }^{1}$, Xiu Tao Sun ${ }^{4}$, Ning Yi Jin ${ }^{5}$ and Xing Kui Si ${ }^{1 *}$

\begin{abstract}
Background: Duck circovirus (DuCV) is a potential immunosuppressive virus that causes feather disorders in young ducks. In this study, DuCV obtained from various species of ducks was investigated by polymerase chain reaction (PCR) in southern and southwestern China (Guangdong, Guangxi and Yunnan provinces) from 2018 to 2019.

Results: A total of 848 bursa samples were collected from dead Mulard, Cherry Valley Pekin, Muscovy and Mallard ducks from duck farms. The positivity rate of DuCV in the total sample was approximately $36.91 \%$. We found that the prevalence of DuCV in Yunnan (43.09\%) was higher than those in Guangxi (34.38\%) and Guangdong (34.4\%). However, the positivity rates of DuCV in the four duck species were not significantly different $(P>0.05)$. Nineteen randomly selected complete viral genomes were sequenced. The complete genomes of the DuCV were 1987 to 1995 nt in length, and were 81.7-99.3\% homologous to the other 57 sequences in GenBank. Phylogenetic analyses based on the complete genomes of 76 DuCVs showed that the 19 novel DuCV sequences from Guangdong and Guangxi provinces mainly belonged to the DuCV-1 and DuCV-2 genetic groups, respectively. However, the two genotype groups coexisted in Yunnan Province. In addition, recombination analysis showed putative recombination sites in 3 strains in Yunnan that originated from strains Guangdong and Guangxi. Interestingly, the epidemiological investigation showed that Mulard ducks, Cherry Valley Pekin ducks and Muscovy ducks more than 4 weeks old were more susceptible to infection with the novel DuCV than ducks less than 4 weeks old.
\end{abstract}

Conclusions: These data provide insight into the molecular epidemiology and genetic diversity of DuCVs circulating in southern and southwestern China for the first time.

Keywords: Duck circovirus, Epidemiological investigation, Phylogeny

\section{Background}

The circovirus family (Circovirus) is a small, round, nonenveloped, single-stranded DNA virus with a circular genome of less than $2 \mathrm{~kb}[1,2]$. The Circovirus genus genome organization is ambisense, meaning that the two main open reading frames (ORFs) are responsible for encoding replication proteins (Rep) and capsid proteins (Cap).

\footnotetext{
*Correspondence: sixingkui_fx@126.com

${ }^{+} \mathrm{Hao}$ Liu, Li Xia Li and Wen Chao Sun contributed equally to this work.

'School of Life Sciences and Engineering, Foshan University, Foshan 528000, China

Full list of author information is available at the end of the article
}

Members include porcine circovirus type 1 (PCV1), porcine circovirus type 2 (PCV2), porcine circovirus type 3 (PCV3), duck circovirus (DuCV), goose circovirus (GoCV), canary circovirus $(\mathrm{CaCV})$, gull circovirus $(\mathrm{GuCV})$ and other circoviruses [3-9].

DuCV was first reported in Germany in 2003 [5, 10]. Since then, DuCV has been reported in a number of countries, including Hungary, China, the US, Italy and South Korea [11-18]. DuCV mainly invades the host's immune system and causes multiple secondary infections in domestic ducks $[5,6,10,17]$. It can cause a variety of clinical symptoms, such as feathering disorders, growth retardation,

(c) The Author(s). 2020 Open Access This article is licensed under a Creative Commons Attribution 4.0 International License, which permits use, sharing, adaptation, distribution and reproduction in any medium or format, as long as you give appropriate credit to the original author(s) and the source, provide a link to the Creative Commons licence, and indicate if changes were made. The images or other third party material in this article are included in the article's Creative Commons licence, unless indicated otherwise in a credit line to the material. If material is not included in the article's Creative Commons licence and your intended use is not permitted by statutory regulation or exceeds the permitted use, you will need to obtain permission directly from the copyright holder. To view a copy of this licence, visit http://creativecommons.org/licenses/by/4.0/ The Creative Commons Public Domain Dedication waiver (http://creativecommons.org/publicdomain/zero/1.0/) applies to the data made available in this article, unless otherwise stated in a credit line to the data. 
low body weight (BW), lymphocyte depletion, necrosis, and histiocytosis in the bursa of Fabricius (BF) [10]. DuCV can be divided into two genotypes, DuCV-1 and DuCV-2, based on the complete genome and cap gene $[17,19]$. Worldwide, there are more reports of DuCV-1 in Germany, Hungary, the US, China, South Korea, and Poland than DuCV-2 reports in Taiwan and China [17]. Recent research has suggested that DuCVs-1 and 2 are mainly concentrated in eastern coastal cities and in migrating wild ducks in China [20]. Epidemics of DuCVs-1 and 2 have been associated with increasingly serious harm to the poultry industry in northern and southern China, including Hebei, Shandong, Fujian and Zhejiang provinces [15, 17, 21]. However, there have been few reports of DuCV epidemic outbreaks in Guangdong, Guangxi and Yunnan provinces. Therefore, this research aimed to better understand the epidemiological and genetic characteristics of DuCV in southern and southwestern China in 2018 and 2019.

\section{Results}

\section{Molecular assays of clinical samples}

In this study, a total of 313 (36.91\%) ducks were positive for DuCV. The positivity rates of DuCV were $34.4 \%(86 / 250)$, $34.38 \%(121 / 352)$ and $43.09 \%(106 / 246)$ in Guangdong, Guangxi and Yunnan provinces, respectively (Table 1). Among the positive samples, 39.47, 31.75 and $43.68 \%$ of Mulard ducks were positive; 30.0, 41.77 and $51.58 \%$ of Cherry Valley Pekin ducks were positive; 40.74, 30.61 and $39.76 \%$ of Muscovy ducks were positive; and 20.69, 33.67 and $42.86 \%$ of Mallard ducks were positive in the three provinces, respectively (Table 1 ). The positivity rate was not significant among the various species of ducks $(P>0.05)$. Among the positive samples, 64 (21.92\%) of 292, 162 (41.65\%) of 389 and $87(52.1 \%)$ of 167 ducks were in the age ranges of 0 to 4,4 to 8 and more than 8 weeks, respectively (Table 2). The DuCV prevalence in ducks more than 8 weeks old was 2.4- and 1.25-times greater than that in ducks 0 to 4 and 4 to 8 weeks old, respectively. The positivity rates were 23.23, 40.12 and $53.03 \%$ in Mulard ducks; 17.74, 42.11 and 51.16\% in Cherry Valley Pekin ducks; and $22.58,45.12$ and $53.33 \%$ in Muscovy ducks aged 0 to 4 weeks, 4 to 8 weeks and more than 8 weeks, respectively. The positivity rates were 23.19 and $41.12 \%$ in Mallard ducks aged 0 to 4 weeks and 4 to 8 weeks; positivity in ducks aged more than 8 week was not recorded in this study (Table 2). The results prompted us to determine that ducks more than 4 weeks old were more susceptible to DuCV than those less than 4 weeks old in Mulard ducks, Cherry Valley Pekin ducks and Muscovy ducks $(P<0.05)$.

\section{Multiple alignment and recombination analysis of DuCV}

Sequence analyses showed that the full genomic lengths of the 19 DuCVs were 1987-1995 nt; the sequences were deposited into GenBank under the accession numbers MK814571-MK814589. Notably, the lengths of the novel DuCV strains GD150501 (MK814571), GD150502 (MK814572), GD180503 (MK814573), GD1 80504 (MK814574), GD190401 (MK814575), GD190402 (MK814576) and GD190403 (MK814577) (1987 bp) were due to a deletion mutation at the 966 site and were different from that of WS-GD01 (1988 bp) from Guangdong. Interestingly, the GX150511 (MK814578), GX190512 (MK814579), GX190509 (MK814580) and GX190510 (MK 814581) strains were 1993 bp; the GX190511 (MK814582) and GX190512 (MK814583) strains were both $1995 \mathrm{bp}$, and there were also differences in the GX11049 (1988 bp) and LZ/11/0 (1994 bp) strains from Guangxi. Moreover, the sequence lengths of the YN180505 (MK814584) and YN180506 (MK814585;1988 bp) as well as the YN190410 (MK814586), YN190411 (MK814587), YN190412 (MK814 588) and YN190415 (MK814589) (1987 bp) sequences were most similar to those of the YN24-2013 (1988 bp) and YN27-2013 (1987 bp) sequences from Yunnan. Multiple sequence alignment of the complete nucleotide sequences of the 19 novel DuCVs showed 81.7-99.3\% homology with the other genome sequences in GenBank and 86.4-99.8\% and 77.5-99.5\% homology with the other ORF 1 and ORF 2 gene sequences in GenBank, respectively. Based on the whole-genome nucleotide sequences analysis, the DuCVs showed multiple mutations compared with Germany and Taiwan lineage strains (data not shown). The sequences MK814571-MK814577, MK814584 and MK814585 shared 6 major variable regions (residues 3 to 15,31 to 64,104 to 124,143 to 159,177 to 213 , and 232 to 238 ) in the cap protein in DuCV-2, consistent with a previous report [6]. The DuCV sequences MK814586-MK814589 had identical substitutions in the DuCV-1 cap protein at positions $9 \mathrm{~A} / \mathrm{P}, 82$ R/H, $106 \mathrm{~S} / \mathrm{T}, 135 \mathrm{~K} / \mathrm{E}, 236 \mathrm{~N} / \mathrm{D}$ (online Technical Appendix Figure S1 A). In addition, MK814588 and MK814589 also had identical substitutions at $28 \mathrm{~L} / \mathrm{V}$ and $216 \mathrm{D} / \mathrm{G}$ in the rep protein. MK814571-MK814577 had identical substitutions in the DuCV-2 rep protein at position $22 \mathrm{E} / \mathrm{D}$ (online Technical Appendix Figure S1 B).

We analyzed 19 full-length sequences, and a putative recombination sites (at nt 989 and 1122) was detected in the genome of recombinant 3 strains (YN190411-China-Yunnan, YN190412-China-Yunnan, and YN190415-China-Yunnan) originating from the strains GD150501-China-Guangdong and GX190512China-Guangxi.

\section{Phylogenetic analysis of DuCVs}

Phylogenetic analyses of the full-length genomes of the 19 novel sequences and 57 reference sequences obtained from GenBank indicated that the DuCVs could be divided into two distinct genetic groups: DuCV-1 (Group 1: Germany lineage) and DuCV-2 (Group 2: Taiwan lineage) (Fig. 1). The DuCVs from Guangdong (MK814571-MK814577) 
Table 1 Details of the bursa samples from different regions in southern and southwestern China

\begin{tabular}{|c|c|c|c|c|c|c|c|c|}
\hline Farms & Province & Breed & Age (weeks) & $\begin{array}{l}\text { Number } \\
\text { tested }\end{array}$ & $\begin{array}{l}\text { Number of } \\
\text { positive ducks }\end{array}$ & $\begin{array}{l}\text { Positive number } \\
\text { (rate \%) of DuCV }\end{array}$ & $\begin{array}{l}\text { Number of Species } \\
\text { ducks positive for } \\
\text { DuCV/(\%) }\end{array}$ & $\begin{array}{l}\text { Number of } \\
\text { ducks positive } \\
\text { for DuCV/(\%) }\end{array}$ \\
\hline 1 & Guangdong & Mulard duck & 2 & 24 & 5 & $20.83 \%$ & 45/(39.47\%) & $86 /(34.4 \%)$ \\
\hline 2 & Guangdong & Mulard duck & 6 & 13 & 5 & $38.46 \%$ & & \\
\hline 3 & Guangdong & Mulard duck & 9 & 34 & 18 & $52.94 \%$ & & \\
\hline 4 & Guangdong, & Mulard duck & 6 & 23 & 9 & $39.13 \%$ & & \\
\hline 5 & Guangdong & Mulard duck & 6 & 20 & 8 & $40.00 \%$ & & \\
\hline 6 & Guangdong & Cherry Valley pekin duck & 2 & 22 & 4 & $18.18 \%$ & $24 /(30 \%)$ & \\
\hline 7 & Guangdong & Cherry Valley pekin duck & 4 & 20 & 4 & $20.00 \%$ & & \\
\hline 8 & Guangdong & Cherry Valley pekin duck & 7 & 38 & 16 & $42.11 \%$ & & \\
\hline 9 & Guangdong & Muscovy duck & 9 & 15 & 8 & $53.33 \%$ & $11 /(40.74 \%)$ & \\
\hline 10 & Guangdong & Muscovy duck & 4 & 12 & 3 & $25.00 \%$ & & \\
\hline 11 & Guangdong, & Mallard duck & 3 & 10 & 2 & $20.00 \%$ & 6/(20.69\%) & \\
\hline 12 & Guangdong, & Mallard duck & 4 & 19 & 4 & $21.05 \%$ & & \\
\hline 13 & Guangxi & Mulard duck & 2 & 42 & 10 & $23.81 \%$ & 40/(31.75\%) & $121 /(34.38 \%)$ \\
\hline 14 & Guangxi & Mulard duck & 5 & 30 & 11 & $36.67 \%$ & & \\
\hline 15 & Guangxi & Mulard duck & 6 & 36 & 15 & $41.67 \%$ & & \\
\hline 16 & Guangxi & Mulard duck & 4 & 18 & 4 & $22.22 \%$ & & \\
\hline 17 & Guangxi & Cherry Valley pekin duck & 9 & 35 & 17 & $48.57 \%$ & $33 /(41.77 \%)$ & \\
\hline 18 & Guangxi & Cherry Valley pekin duck & 10 & 24 & 13 & $54.17 \%$ & & \\
\hline 19 & Guangxi & Cherry Valley pekin duck & 2 & 20 & 3 & $15.00 \%$ & & \\
\hline 20 & Guangxi & Muscovy duck & 4 & 19 & 4 & $21.05 \%$ & 15/(30.61\%) & \\
\hline 21 & Guangxi & Muscovy duck & 5 & 30 & 11 & $36.67 \%$ & & \\
\hline 22 & Guangxi & Mallard duck & 4 & 25 & 7 & $28.00 \%$ & 33/(33.67\%) & \\
\hline 23 & Guangxi & Mallard duck & 5 & 30 & 12 & $40.00 \%$ & & \\
\hline 24 & Guangxi & Mallard duck & 5 & 28 & 11 & $39.29 \%$ & & \\
\hline 25 & Guangxi & Mallard duck & 3 & 15 & 3 & $20.00 \%$ & & \\
\hline 26 & Yunnan & Mulard duck & 7 & 22 & 9 & $40.91 \%$ & $38 /(43.68 \%)$ & 106/(43.09\%) \\
\hline 27 & Yunnan & Mulard duck & 7 & 18 & 8 & $44.44 \%$ & & \\
\hline 28 & Yunnan & Mulard duck & 4 & 15 & 4 & $26.67 \%$ & & \\
\hline 29 & Yunnan & Mulard duck & 9 & 32 & 17 & $53.13 \%$ & & \\
\hline 30 & Yunnan & Cherry Valley pekin duck & 9 & 27 & 14 & $51.85 \%$ & $14 /(51.85 \%)$ & \\
\hline 31 & Yunnan & Muscovy duck & 6 & 22 & 10 & $45.45 \%$ & 33/(39.76\%) & \\
\hline 32 & Yunnan & Muscovy duck & 8 & 30 & 16 & $53.33 \%$ & & \\
\hline 33 & Yunnan & Muscovy duck & 3 & 31 & 7 & $22.58 \%$ & & \\
\hline 34 & Yunnan & Mallard duck & 6 & 27 & 12 & $44.44 \%$ & $21 /(42.86 \%)$ & \\
\hline 35 & Yunnan & Mallard duck & 5 & 22 & 9 & $40.91 \%$ & & \\
\hline Total & Three province & ducks & & 848 & 313 & & & $313 /(36.91 \%)$ \\
\hline
\end{tabular}

formed a distinct clade within DuCV-2, while the DuCVs from Yunnan (MK814584 and MK814585) clustered in one branch. The DuCVs from Guangxi (MK814586MK814589) and Yunnan (MK814578-MK814583) clustered in another branch within DuCV-1. The results of the phylogenetic analysis indicated that two genetic groups of DuCVs had caused epidemics in ducks in three provinces.

\section{Discussion}

As stated in previous reports, DuCV may be an immunosuppressive virus that may increase the pathogenicity of coinfecting agents. The classic symptoms are generally considered to be feather disorders, poor body condition and low weight for age. The virus can persist in the thymus, liver, spleen, kidney and BF, and among 


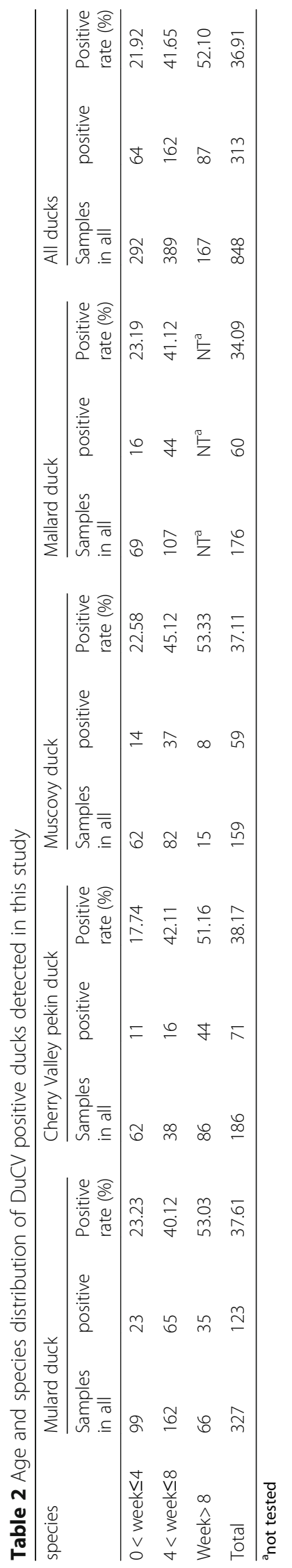




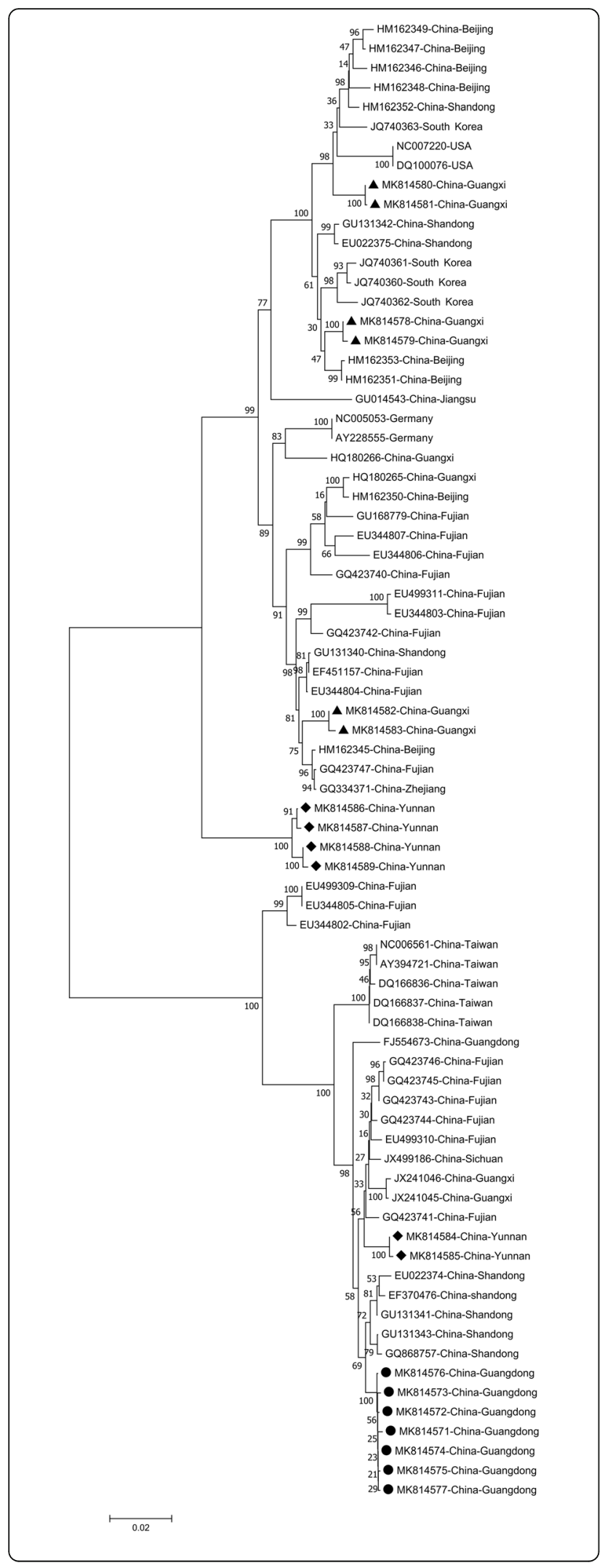

Fig. 1 Phylogenetic analysis of novel duck circovirus (DuCV) wholegenome sequences using the neighbor-joining (NJ) method with 1000 bootstrap replications (Mega 7.0). DuCV-1-duck circovirus genotype 1, DuCV-2-duck circovirus genotype 2. The sequence of the circovirus detected in this study is marked with a symbol. DuCV strains from Guangdong are indicated with circles, those from Guangxi with a triangle, and those from Yunnan with a square. The other sequences were obtained from GenBank, and the accession numbers of these sequences are included in the phylogenetic tree

these organs, the bursa is the most predominant location for circovirus replication $[5,11,13,15,16,22,23]$. There are no existing vaccines or drugs to cure or prevent $\mathrm{DuCV}$, resulting in serious economic and production losses in the poultry industry. The prevalence of DuCV has been extensively reported in multiple species of ducks and from many provinces and cities in China [20,24].

In this study, we aimed to elucidate the epidemiology of DuCVs circulating in southern and southwestern China. PCR methods were used in a molecular epidemiological investigation of $\mathrm{DuCV}$ in the $\mathrm{BF}$ of dead ducks, and the results confirmed a high overall $\mathrm{DuCV}$ infection rate in four species of ducks from Guangdong, Guangxi and Yunnan Province. However, the positivity rates of $\mathrm{DuCV}$ by species and province were not significantly different. These results were similar to previous reports in which DuCV infection was ubiquitous, showing no species dependency $[15,18]$. In addition, the positivity rates showed that Mulard ducks, Cherry Valley Pekin ducks and Muscovy ducks more than 4 weeks old were more susceptible than ducks less than 4 weeks old, consistent with a previous report [15].

Similar to those of other members of the DuCV family, the genomic sequence of the $19 \mathrm{DuCVs}$ contained three major ORFs: ORF 1, ORF 2 and ORF 3 [22, 25]. According to the phylogenetic analyses of the complete genomes, the DuCV in this study belonged to two genotypes [6]. Compared to previous reports, the 19 DuCVs detected in this study belonged to the Group 1 Germany lineage or Group 2 Taiwan lineage [15]. Based on these results, we confirmed that among these 19 DuCVs, Group 1 was prevalent in Guangdong, Group 2 was prevalent in Guangxi and both groups were prevalent in Yunnan province. Interestingly, there were differences between the DuCVs identified in this study and other DuCVs from previous reports, and the genome lengths of the novel sequences GD150501, GD150502 GD180503, GD180504, GD190401, GD190402 and GD190403 were all $1987 \mathrm{nt}$, which is the shortest observed DuCV genome on record [15, 20, 24, 26]. When the sequences were compared with the other genomes in GenBank, it became clear that there was a deletion at the 966 site in the intragenic region in 7 DuCVs from Guangdong, and other DuCVs also showed multiple 
mutations in the complete sequences. This suggests that there are mutations in the novel DuCV gene in the poultry of southern and southwestern China. In addition, we found that potential recombination events occurred in 3 strains from Yunnan, originating in strains from Guangdong and Guangxi. Geographically, Guangxi, Guangdong, and Yunnan are adjacent to each other, and the DuCV sequences were similar, leading to recombination events occurring in Yunnan. Our hypothesis is that virus transmission may have occurred due to trade within the province. A limitation of this study was that we collected only dead ducks; and novel DuCV should be collected and detected in Mallard ducks in the future. Additionally, other virus genotypes may infect ducks but produce no clinical symptoms. Therefore, we cannot rule out the existence of other genotypes in this region, but whether they cause clinical symptoms requires further investigation.

\section{Conclusion}

This study will help elucidate the epidemiological and molecular characteristics of $\mathrm{DuCV}$ in southern and southwestern China. The acquired data suggest that DuCVs are highly prevalent and that there were two distinct genetic groups of novel DuCV in 4 species of ducks in 2018 and 2019. The novel DuCVs can infect different breeds of ducks, and ducks over 4 weeks old were the most susceptible. We were the first to report variations in the complete genome lengths of and recombination in a novel DuCV in southern and southwestern China $[15,20,24-26]$. DuCVs in additional species of ducks remain to be detected, and no wild bird samples were collected. In the future, we need to investigate additional species in poultry and wild-birds to determine the exact origin and analyze the transmission and pathogenesis of DuCVs to aid in the development of more effective vaccines against various DuCVs.

\section{Methods}

\section{Sample collection}

A total of $848 \mathrm{BF}$ samples were collected from four species of ducks ranging from 2 to 10 weeks in age that presented clinical symptoms (feathering disorders, growth retardation and general sickness) and later died. Upon death, BF extraction was conducted according to standard care guidelines. The BFs from 327 Mulard ducks, 186 Cherry Valley Pekin ducks, 159 Muscovy ducks, and 176 Mallard ducks from 53 duck farms in Guangdong (20), Yunnan (17) and Guangxi (16) provinces in China from 2018 to 2019 were removed and sectioned (Table 1).

\section{PCR detection of DuCV}

Total viral DNA was extracted from $0.1 \mathrm{~g}$ of BF samples by a previously described method [24]. The DNA was used to detect DuCV pathogens by PCR using the common DuCV detection primers F1: (5'-ATATTATTAC CGGCGC (C/T) TG-3') and R1: (TCAGGAATCCCTG (A/C) AGGTGA-3') [12]. The complete genome was amplified using the primers F2: (5'-TCCGGATCCGAA AAATCCAAATAC-3') and R2: (5'-CCCGGATCCG GAACTGGACCAAC-3') [17]. The specific PCR products were purified using an agarose gel DNA purification kit and cloned using a pMD18-T kit (Takara, Dalian, China). The positive recombinant clones from three independent amplifications of every strain were Sanger sequenced in both directions (Sangon Biotech, Shanghai, China).

\section{Statistical analysis}

All one-sample $t$ tests were performed using GraphPad Prism 6 (GraphPad Software, Inc.), and two-tailed $P<0.05$ was defined as statistically significant.

\section{Sequence analysis}

Genetic variation and phylogenetic relationships with the complete genomes from $76 \mathrm{DuCV}$ sequences available in GenBank were analyzed (online Technical Appendix Table S1). Nucleotide sequence alignments and homology comparisons were made using the Clustal W method in the MegAlign program (DNASTAR, Madison, USA). The neighbor-joining (NJ) method was used to generate phylogenetic trees based on the aligned nucleotide sequences in MEGA v7.0 [4, 27]. To detect putative recombination breakpoints in the $\mathrm{DuCV}$ genome and to identify sequences that possibly originated from a recombination event, the RDP 4.24 program was used [28].

\section{Supplementary information}

Supplementary information accompanies this paper at https://doi.org/10. 1186/s12917-020-02301-X.

Additional file 1: Figure S1. Deduced amino acid sequence comparisons of the 19 DuCV strains in Cap (A) and Rep (B) protein. Table S1. Details of DuCV isolates used in this study and other isolates available in GenBank.

\section{Abbreviations}

BF: Bursa of Fabricius; Cap: Capsid protein; DuCV: Duck circovirus; DuCV1: Duck circovirus genotype 1; DuCV-2: Duck circovirus genotype 2; NJ: Neighbor-joining; ORF: Open reading frame; Rep: Replication protein

\section{Acknowledgments}

We thank Professors Hui-jun Lu at the Military Veterinary Institute, Academy of Military Medical Sciences, and Quan Liu at the School of Life Sciences and Engineering, Foshan University, for their discussions and suggestions.

\section{Authors' contributions}

$\mathrm{HL}$ performed the research, analyzed the data, and wrote the manuscript. LXL, WCS, NS and XTS participated in sample collection and research testing. NYJ and XKS designed the research. All authors read and approved the final manuscript. 


\section{Funding}

This work was supported by the National Natural Sciences Foundation of China (31802199), the Key Laboratory for Preventive Research of Emerging Animal Diseases in Foshan University (KLPREAD201801-07). The funding body was solely involved in infusion and had no role in the design of the study; in the collection, analysis, or interpretation of the data; or in writing the manuscript.

\section{Availability of data and materials}

The datasets used and/or analyzed during the current study are available from the corresponding author upon reasonable request.

\section{Ethics approval and consent to participate}

The duck samples used in this study were obtained from the Military Veterinary Institute, Academy of Military Medical Sciences, and the experiment was approved by the Institutional Animal Care and Use Committee (IACUC) of the Chinese Academy of Military Medical Science, Changchun, China (10ZDGG007). Written consent for the use of duck samples before participation in the study was obtained from the duck's owners.

\section{Consent for publication}

Not applicable.

\section{Competing interests}

The authors declare that they have no competing interests.

\section{Author details}

${ }^{1}$ School of Life Sciences and Engineering, Foshan University, Foshan 528000, China. ${ }^{2}$ Jilin Wildlife Rescue and Rehabilitation Center, Forestry Department of Jilin Province, Changchun 130122, China. ${ }^{3}$ Institute of Virology, Wenzhou University, Wenzhou 325035, China. ${ }^{4}$ Honghe Animal Disease Prevention and Control Center, Mengzi 661000, China. ${ }^{5}$ Military Veterinary Institute, Academy of Military Medical Sciences, Changchun 130122, China.

Received: 16 June 2019 Accepted: 28 February 2020

Published online: 05 March 2020

\section{References}

1. Hughes AL, Piontkivska $\mathrm{H}$. Nucleotide sequence polymorphism in circoviruses. Infect Genet Evol. 2008;8(2):130-8

2. Todd D. Avian circovirus diseases: lessons for the study of PMWS. Vet Microbiol. 2004;98(2):169-74.

3. He W, Zhao J, Xing G, Li G, Wang R, Wang Z, Zhang C, Franzo G, Su S, Zhou J. Genetic analysis and evolutionary changes of porcine circovirus 2. Mol Phylogenet Evol. 2019:139:106520.

4. Li G, He W, Zhu H, Bi Y, Wang R, Xing G, Zhang C, Zhou J, Yuen KY, Gao GF, et al. Origin, genetic diversity, and evolutionary dynamics of novel porcine Circovirus 3. Adv Sci (Weinh). 2018;5(9):1800275.

5. Hattermann K, Schmitt C, Soike D, Mankertz A. Cloning and sequencing of duck circovirus (DuCV). Arch Virol. 2003;148(12):2471-80.

6. Wang D, Xie X, Zhang D, Ma G, Wang X, Zhang D. Detection of duck circovirus in China: a proposal on genotype classification. Vet Microbiol. 2011;147(3-4):410-5

7. Mankertz A, Hattermann K, Ehlers B, Soike D. Cloning and sequencing of columbid circovirus (coCV), a new circovirus from pigeons. Arch Virol. 2000; 145(12):2469-79.

8. Todd D, Scott AN, Fringuelli E, Shivraprasad HL, Gavier-Widen D, Smyth JA. Molecular characterization of novel circoviruses from finch and gull. Avian Pathol. 2007;36(1):75-81.

9. Soike $D$, Kohler B, Albrecht K. A circovirus-like infection in geese related to a runting syndrome. Avian Pathol. 1999:28(2):199-202.

10. Soike D, Albrecht K, Hattermann K, Schmitt C, Mankertz A. Novel circovirus in mulard ducks with developmental and feathering disorders. Vet Rec. 2004;154(25):792-3.

11. Fringuelli $E$, Scott AN, Beckett A, Mckillen J, Smyth JA, Palya V, Glavits R, Ivanics E, Mankertz A, Franciosini MP, et al. Diagnosis of duck circovirus infections by conventional and real-time polymerase chain reaction tests. Avian Pathol. 2005;34(6):495-500.
12. Chen CL, Wang PX, Lee MS, Shien JH, Shien HK, Ou SJ, Chen CH, Chang PC. Development of a polymerase chain reaction procedure for detection and differentiation of duck and goose circovirus. Avian Dis. 2006;50(1):92-5.

13. Banda A, Galloway-Haskins RI, Sandhu TS, Schat KA. Genetic analysis of a duck circovirus detected in commercial Pekin ducks in New York. Avian Dis. 2007:51(1):90-5.

14. Liu SN, Zhang XX, Zou JF, Xie ZJ, Zhu YL, Zhao Q, Zhou EM, Jiang SJ. Development of an indirect ELISA for the detection of duck circovirus infection in duck flocks. Vet Microbiol. 2010;145(1-2):41-6.

15. Wan CH, Fu GH, Shi SH, Cheng LF, Chen HM, Peng CX, Lin S, Huang Y. Epidemiological investigation and genome analysis of duck circovirus in southern China. Virol Sin. 2011;26(5):289-96.

16. Zhang $X$, Jiang $S$, Wu J, Zhao Q, Sun Y, Kong Y, Li X, Yao M, Chai T. An investigation of duck circovirus and co-infection in Cherry Valley ducks in Shandong Province, China. Vet Microbiol. 2009;133(3):252-6.

17. Zhang Z, Jia R, Lu Y, Wang M, Zhu D, Chen S, Yin Z, Chen X, Cheng A. Identification, genotyping, and molecular evolution analysis of duck circovirus. Gene. 2013;529(2):288-95.

18. Cha SY, Kang M, Cho JG, Jang HK. Genetic analysis of duck circovirus in Pekin ducks from South Korea. Poult Sci. 2013;92(11):2886-91.

19. Wen H, Wu Y, Yang C, Zhang X, Lian C, Chen H, Han L. Comments on duck circovirus (DuCV) genotype definition. Gene. 2014;538(1):207-8.

20. Niu X, Liu L, Han C, Li J, Zeng X. First findings of duck circovirus in migrating wild ducks in China. Vet Microbiol. 2018;216:67-71.

21. Fu GH, Cheng LF, Shi SH, Peng CX, Chen HM, Huang Y. Genome cloning and sequence analysis of duck circovirus. Bing Du Xue Bao. 2008;24(2):138-43.

22. Xiang QW, Wang X, Xie ZJ, Sun YN, Zhu YL, Wang SJ, Liu HJ, Jiang SJ. ORF3 of duck circovirus: a novel protein with apoptotic activity. Vet Microbiol. 2012;159(1-2):251-6.

23. Matczuk AK, Krawiec M, Wieliczko A. A new duck circovirus sequence, detected in velvet scoter (Melanitta fusca) supports great diversity among this species of virus. Virol J. 2015;12:121.

24. Li P, Li J, Zhang R, Chen J, Wang W, Lan J, Xie Z, Jiang S. Duck "beak atrophy and dwarfism syndrome" disease complex: interplay of novel goose parvovirus-related virus and duck circovirus? Transbound Emerg Dis. 2018; 65(2):345-51.

25. Zhang XX, Liu SN, Xie ZJ, Kong YB, Jiang SJ. Complete genome sequence analysis of duck circovirus strains from Cherry Valley duck. Virol Sin. 2012; 27(3):154-64.

26. Xie L, Xie Z, Zhao G, Liu J, Pang Y, Deng X, Xie Z, Fan Q. Complete genome sequence analysis of a duck circovirus from Guangxi pockmark ducks. J Virol. 2012:86(23):13136.

27. Tamura K, Stecher G, Peterson D, Filipski A, Kumar S. MEGA6: Molecular Evolutionary Genetics Analysis version 6.0. Mol Biol Evol. 2013;30(12):2725-9.

28. Martin DP, Lemey P, Lott M, Moulton V, Posada D, Lefeuvre P. RDP3: a flexible and fast computer program for analyzing recombination. Bioinformatics. 2010;26(19):2462-3.

\section{Publisher's Note}

Springer Nature remains neutral with regard to jurisdictional claims in published maps and institutional affiliations.

Ready to submit your research? Choose BMC and benefit from:

- fast, convenient online submission

- thorough peer review by experienced researchers in your field

- rapid publication on acceptance

- support for research data, including large and complex data types

- gold Open Access which fosters wider collaboration and increased citations

- maximum visibility for your research: over $100 \mathrm{M}$ website views per year

At $\mathrm{BMC}$, research is always in progress.

Learn more biomedcentral.com/submission 